\title{
Dental Caries Prediction Based on a Survey of the Oral Health Epidemiology among the Geriatric Residents of Liaoning, China
}

\author{
Lu Liu, ${ }^{1,2}$ Wei Wu, ${ }^{2}$ Si-yu Zhang, ${ }^{1}$ Kai-qiang Zhang, ${ }^{1}$ Jian Li, ${ }^{1}$ Yang Liu, ${ }^{1}$ and Zhi-hua Yin $\mathbb{D}^{2}$ \\ ${ }^{1}$ Department of Preventive Dentistry, School and Hospital of Stomatology, China Medical University, Liaoning Provincial Key \\ Laboratory of Oral Diseases, Shenyang 110002, China \\ ${ }^{2}$ Department of Epidemiology, School of Public Health, China Medical University, Shenyang 110122, China
}

Correspondence should be addressed to Zhi-hua Yin; zhyin@cmu.edu.cn

Received 22 April 2020; Revised 12 November 2020; Accepted 26 November 2020; Published 7 December 2020

Academic Editor: Min Hui Li

Copyright @ $2020 \mathrm{Lu}$ Liu et al. This is an open access article distributed under the Creative Commons Attribution License, which permits unrestricted use, distribution, and reproduction in any medium, provided the original work is properly cited.

Background. Dental caries is one of the most common chronic diseases observed in elderly patients. The development of preventive strategies for dental caries in elderly individuals is vital. Objective. The objective of the present study was to construct a generalized regression neural network (GRNN) prediction model for the risk assessment of dental caries among the geriatric residents of Liaoning, China. Methods. A stratified equal-capacity random sampling method was used to randomly select 1144 elderly (65-74 years) residents (gender ratio 1:1) of Liaoning, China. Data for the oral assessment, including caries characteristics, and questionnaire survey from each participant were collected. Multivariate logistic regression analysis was then performed to identify the independent predictors. GRNN was applied to establish a prediction model for dental caries. The accuracy of the unconditional logistic regression and the GRNN early warning model was compared. Results. A total of 1144 patients fulfilled the requirements and completed the questionnaires. The caries rate was $68.5 \%$, and the main associated factors were toothache history, residence area, smoking, and drinking. We randomly divided the data for the 1144 participants into a training set (915 cases) and a test set (229 cases). The optimal smoothing factor was 0.7 , and the area under the receiver operating characteristic curve for the GRNN model was 0.626 (95\% confidence interval, 0.544 to 0.708 ), with a $P$ value of 0.002 . In terms of consistency, sensitivity, and specificity, the GRNN model was better than the traditional unconditional multivariate logistic regression model. Conclusion. Geriatric (65-74 years) residents of Liaoning, China, have a high rate of dental caries. Residents with a history of toothache and smoking habits are more susceptible to the disease. The GRNN early warning model is an accurate and meaningful tool for screening, early diagnosis, and treatment planning for geriatric individuals with a high risk of caries.

\section{Introduction}

Dental caries (tooth decay) is one of the most common chronic diseases observed in elderly populations [1-4]. Dental caries is more prevalent in elderly individuals who have a higher risk of losing teeth due to caries than other groups of people $[4,5]$. Additionally, poor oral health can have a significant impact on the quality of life for patients and increase the risk of associated systemic chronic diseases [6-8]. Therefore, the development of preventive strategies for dental caries is vital.

China has the largest aging population in the world, accounting for $20 \%$ of the global total [9]. However, being a developing country, China has a relative shortage of oral healthcare facilities and medical insurance services are not universal throughout the country [10]. Moreover, elderly individuals have limited access to oral healthcare services, remarkably potentiating the burden of senile dental caries. Four national oral health epidemiological surveys have been conducted periodically (in 1983, 1995, 2005, and 2015) to determine the prevalence and regularity of senile dental caries in China. The caries prevalence and average decayedmissing-filled teeth (DMFT) indices in the Chinese elderly population were reported to be $98.4 \%$ and $14.65 \%$, respectively [11, 12]. Additionally, most cases of dental caries $(>60 \%)$ were concentrated in $20 \%$ of participants who were at a high risk of developing the disease [13]. These findings emphasized the urgent need for the prevention and timely 
management of dental caries among geriatric residents in China. Although Liaoning province is located in the center of northeast China economic zone, the burden of oral diseases is significant among the residents, corresponding to their lower level of awareness about oral health [14]. Identification of the high-risk groups among the geriatric population and management of the disease with targeted therapy provided accordingly can be beneficial to effectively control the development of dental caries.

Current prediction methods for dental caries are based on traditional logistic regression models. However, due to the limitations of the principles of regression, such as that the variables must satisfy the criteria for independence and other assumptions, the logistic regression model cannot address the collinearity problem between variables. Therefore, the application of the logistic regression model for the prediction of caries in the elderly population has its limitations.

An artificial neural network (ANN) is an information processing system that mimics the structure and function of the biological brain [15]. There are four main neural network models currently used in practice: the back-propagation neural network (BPNN), generalized regression neural network (GRNN), fuzzy neural network (FNN), and probabilistic neural network (PNN) [16]. GRNN is a four-layer forward network consisting of an input layer, a hidden layer, a summation layer, and a division layer and has a strong nonlinear mapping ability and accurate prediction capacity. Compared to other models, GRNN has a number of advantages such as approximating ability, classification ability, and learning speed [17]. In addition, GRNN converges on the optimal regression surface with large-sample aggregation that has a reasonable extrapolation and good prediction effects when the sample data is lacking [18]. Therefore, GRNN adapts to the high complexity, nonlinearity, and uncertainty associated with the occurrence and development of dental caries in the elderly population. There are only a few reports on the application of GRNN for disease prediction in the field of stomatology. Zakrzewska et al. used GRNN to study the fluoride distribution in the mandible and teeth [19]. To the best of our knowledge, no published reports describe the utilization of GRNN to establish a prediction model for dental caries in the elderly population. Therefore, the objective of the present study was to construct a GRNN prediction model for the risk assessment of dental caries among the geriatric residents of Liaoning, China. For this purpose, data from a random sample survey of the oral health epidemiology of the elderly population were analyzed.

\section{Materials and Methods}

The present cross-sectional survey study was approved by the institutional review board of the School and Hospital of Stomatology, China Medical University, Liaoning Provincial Key Laboratory of Oral Diseases, Shenyang, China. The data were collected from May to December 2015. Each participant underwent an oral health examination and completed a faceto-face oral questionnaire survey. The data collection and analysis were performed in 2018. The project followed the ethical guidelines provided by the World Medical Association Declaration of Helsinki (2013).

2.1. Study Participants. The anticipated sample size was determined based on the sixth edition of "Stomatological Preventive Medicine" [20]. The sample content for the present cross-sectional survey was calculated using the following formula: $N=k \times(1-P) / P$. In this formula, $N$ is the sample size, $k$ is defined based on the allowable error for the research project, and $P$ is the expected prevalence of dental caries. With an allowable error of $10 \%$, a $k$ value determined as 400 according to the allowable error size, and an expected prevalence of $67.5 \%$ for dental caries $(P)$ (the incidence of senile caries in the three northeastern provinces in 2013) [21], the sample size $(N)$ required was $N=400 \times$ $32.5 / 67.5=193$. To ensure an adequate and effective sample size and avoid loss of follow-up or rejection, the recruitment sample size was expanded.

A stratified equal-capacity random sampling method was used to randomly select 1168 geriatrics (65-74 years; gender ratio $1: 1)$ among the urban and rural residents of the Liaoning province, China. The eligibility criteria included age (65-74 years), willingness to cooperate with clinical oral examinations, and residing in the locality of the survey for more than six months. The elderly who were over 75 years old were excluded from the study as they were unable to cooperate with an oral examination, illiterate, or unable to answer the questions in the questionnaire were excluded from the survey. A total of 1144 participants met the required criteria and completed the questionnaires. Informed consent was obtained from each participant (see study diagram in Figure 1).

2.2. Assessment of Study Participants. All 1144 study participants received a comprehensive dental examination at the study clinic. The examinations were done by a team of calibrated and trained dentists (Liu, Zhang and Li, Zhang) and included three components:

(1) General oral assessment, including dentition status and caries assessment

(2) Caries characteristics, including the number of carious lesions and category (crown caries and/or root caries). According to the World Health Organization (WHO), crown caries refers to the formation of an obvious cavitation, enamel destruction, or clear lesions with a softening bottom or wall of the tooth surface, detected during Community Periodontal Index (CPI) probing. Root caries refers to the exposed root showing a soft or leathery lesion, detected with CPI probes [22]. The full list of the related variables is given in Appendix II

(3) Through face-to-face interviews, a questionnaire was used to compile the patients' data regarding their age, gender, oral hygiene habits, fluoride toothpaste use, eating pattern, household registration type, oral health behavior, oral medical treatment, education 


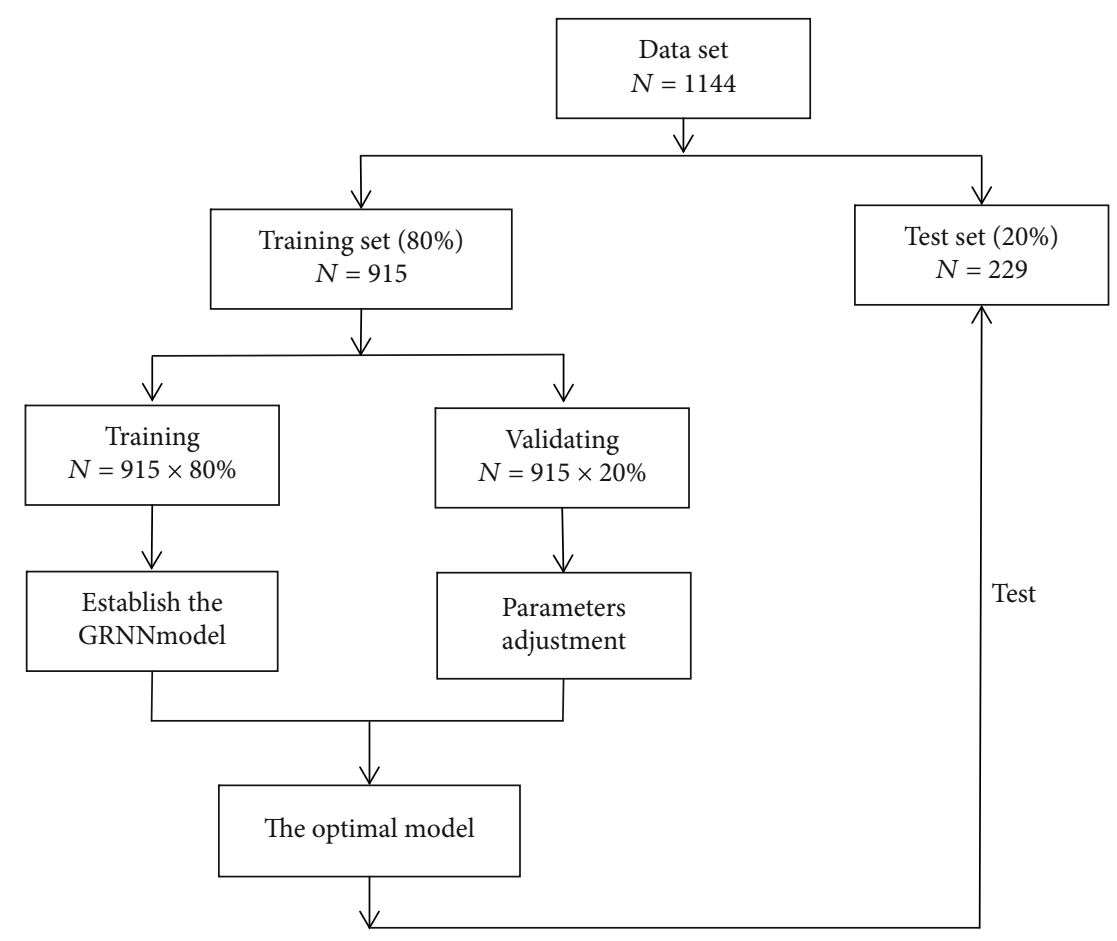

FIGURE 1: Flowchart representing the development of the generalized regression neural network (GRNN) model.

level, and dental health knowledge. See more details in Appendix II

The definitions of predictors were as follows: (a) eating candy frequently: the frequency of eating sweet foods (such as sugary or carbonated drinks, fresh fruits, cookies, cakes, candies, chocolates, sugary milk, and yogurt) was greater than or equal to twice per week. (b) Smoking/drinking habit: elderly participants who had a habit of smoking or drinking daily or weekly were defined as having a habit of smoking or drinking. (c) Use of toothpaste/toothpick/floss was defined as frequent if the usage frequency was greater than twice a week.

2.3. Quality Control. The examination standards for dental caries detection in the participants were based on the WHO Basic Methods for Oral Health Survey [22]. One examiner and three questionnaire investigators, who were senior dental specialists, conducted the investigation. All investigators were trained before the start of the formal investigation. The four-member research team conducted a pilot study on a sample population in Liaoning province, China. According to the standard conformance test, the kappa values between the investigators were $\sim 0.85$. The on-site formal inspection facilities were the same as those used for the pilot research and included a mobile dental examination chair, illumination lamp, and CPI probes.

2.4. Statistical Analysis. Continuous variables were summarized with descriptive statistics in the form of mean and standard deviations (SD). The categorical variables were described as counts and percentages. Univariable Pearson's chi-squared test was used to screen out meaningful factors
$(P<0.05)$ that may affect the occurrence of dental caries. Multivariate logistics regression analysis was performed to identify the independent predictors using variables that had a statistical association with the occurrence of dental caries. The odds ratio (OR) and 95\% confidence interval (CI) were used to describe the associations.

We randomly divided the data for the 1144 participants into two parts: $80 \%$ (915 cases) were included in the training set for the establishment of unconditional logistic regression and the GRNN early warning model, and the remaining $20 \%$ (229 cases) were used as the test set for model prediction.

Based on the one-way chi-square data for the training set, statistically significant variables were included in the unconditional logistic regression model using the forward method to establish a multifactor predictive model. The unconditional multivariate logistic regression model was then used to predict whether the subjects in the test set (229 cases) had dental caries.

The statistically significant variables from the chi-square test were used as the input, and the outcome variable (the presence of dental caries) was the output. The GRNN early warning model was established with the neural network toolbox using the MATLAB software (MathWorks, Natick, MA). Afterward, 20\% of participants (183 out of 915) were randomly selected from the training set as a special test set to find the optimal smoothing factor for GRNN. The smoothing factor was determined using the method proposed by Sprecht [23]. We increased the unit quantity (0.1) in increments of 0.1 and obtained the respective predicted value for the test set. The optimal smoothing factor was set when the mean square error between the test set predicted value and the sample measured value was the smallest. The receiver operating characteristic (ROC) curve for the model prediction results 
TABLE 1: The demographic and lifestyle characteristics of the study participants $(n=1144)$.

\begin{tabular}{|c|c|}
\hline Characteristic & Value \\
\hline \multicolumn{2}{|l|}{ Demographic characteristics } \\
\hline Age $($ mean $\pm S D)$, years & $68.29 \pm 3.122$ \\
\hline Female, $n(\%)$ & $576(50.3)$ \\
\hline The number of people with caries, $n(\%)$ & $784(68.5)$ \\
\hline \multicolumn{2}{|l|}{ Residence area } \\
\hline Urban, $n(\%)$ & $573(50.1)$ \\
\hline Rural, $n(\%)$ & $571(49.9)$ \\
\hline \multicolumn{2}{|c|}{ Use of a removable upper jaw dental prosthesis } \\
\hline Yes, $n(\%)$ & $156(13.6)$ \\
\hline No, $n(\%)$ & $988(86.4)$ \\
\hline \multicolumn{2}{|c|}{ Use of a removable lower jaw dental prosthesis } \\
\hline Yes, $n(\%)$ & $123(10.8)$ \\
\hline No, $n(\%)$ & $1021(89.2)$ \\
\hline \multicolumn{2}{|l|}{ Number of true teeth } \\
\hline$>n ?$ & $730(63.8)$ \\
\hline$\leq n$ & $414(36.2)$ \\
\hline \multicolumn{2}{|l|}{ Dental insurance } \\
\hline Yes, $n(\%)$ & $144(12.6)$ \\
\hline No, $n(\%)$ & $1000(87.4)$ \\
\hline \multicolumn{2}{|l|}{ Having toothache in previous years } \\
\hline Yes, $n(\%)$ & $535(46.8)$ \\
\hline No, $n(\%)$ & $609(53.2)$ \\
\hline \multicolumn{2}{|l|}{ Lifestyle } \\
\hline \multicolumn{2}{|l|}{ Smoking } \\
\hline Yes, $n(\%)$ & $737(64.4)$ \\
\hline No, $n(\%)$ & $407(35.6)$ \\
\hline \multicolumn{2}{|l|}{ Drinking alcohol } \\
\hline Yes, $n(\%)$ & $907(79.3)$ \\
\hline No, $n(\%)$ & $237(20.7)$ \\
\hline \multicolumn{2}{|l|}{ Eating candy frequently } \\
\hline Yes, $n(\%)$ & $72(6.3)$ \\
\hline No, $n(\%)$ & $1072(93.7)$ \\
\hline \multicolumn{2}{|l|}{ Drinking carbonated beverages frequently } \\
\hline Yes, $n(\%)$ & $32(2.8)$ \\
\hline No, $n(\%)$ & $1112(97.2)$ \\
\hline \multicolumn{2}{|l|}{ Domestic water access } \\
\hline Tap water, $n(\%)$ & $732(64.0)$ \\
\hline Not tap water, $n(\%)$ & $412(36.0)$ \\
\hline \multicolumn{2}{|l|}{ Use of toothpick } \\
\hline Yes, $n(\%)$ & $720(62.9)$ \\
\hline No, $n(\%)$ & $424(37.1)$ \\
\hline \multicolumn{2}{|l|}{ Use of dental floss } \\
\hline Yes, $n(\%)$ & $4(0.3)$ \\
\hline No, $n(\%)$ & $1140(99.7)$ \\
\hline \multicolumn{2}{|l|}{ Use of fluoride toothpaste } \\
\hline Yes, $n(\%)$ & $338(29.5)$ \\
\hline No, $n(\%)$ & $806(70.5)$ \\
\hline
\end{tabular}

TABle 1: Continued.

\begin{tabular}{|c|c|}
\hline Characteristic & Value \\
\hline \multicolumn{2}{|c|}{ Beliefs and behaviors } \\
\hline \multicolumn{2}{|c|}{ Healthcare service utilization } \\
\hline Yes, $n(\%)$ & $749(65.5)$ \\
\hline No, $n(\%)$ & $395(34.5)$ \\
\hline \multicolumn{2}{|c|}{ Believing oral health has an impact on eating } \\
\hline Yes, $n(\%)$ & $641(56.0)$ \\
\hline No, $n(\%)$ & $503(44.0)$ \\
\hline \multicolumn{2}{|c|}{ Visiting dental clinic for caries without pain } \\
\hline Yes, $n(\%)$ & $296(25.9)$ \\
\hline No, $n(\%)$ & $848(74.1)$ \\
\hline \multicolumn{2}{|c|}{ Visiting dental clinic in the past year } \\
\hline Yes, $n(\%)$ & $193(16.9)$ \\
\hline No, $n(\%)$ & $951(83.1)$ \\
\hline \multicolumn{2}{|c|}{ Visiting dental clinic for toothache } \\
\hline Yes, $n(\%)$ & $309(27.0)$ \\
\hline No, $n(\%)$ & $835(73.0)$ \\
\hline \multicolumn{2}{|c|}{ Self-oral health assessment } \\
\hline Not good, $n(\%)$ & $447(39.1)$ \\
\hline Good, $n(\%)$ & $697(60.9)$ \\
\hline \multicolumn{2}{|c|}{ Self-oral hygiene assessment } \\
\hline Not good, $n(\%)$ & $258(22.6)$ \\
\hline Good, $n(\%)$ & $886(77.4)$ \\
\hline
\end{tabular}

was plotted. The model prediction probability value corresponding to the maximum value of Youden's index (sensitivity + specificity-1) during training was set as the best cut-off value for the present study. The comparison between the unconditional logistic regression and GRNN models was performed according to the statistical analysis of consistency, sensitivity, specificity, and area under the ROC curve. Finally, the optimal cut-off value for the predictive probability of the logistic regression model in the present study was determined to be 0.606 , with a corresponding Youden's index of 0.370 . The optimal cut-off value for the GRNN model's predictive probability was 0.680 , with a corresponding Youden's index of 0.638 . $P$ values less than 0.05 were considered statistically significant for all tests. Statistical analyses of all the data except the GRNN model were performed using SPSS 22.0 (BMI Corporation, Chicago, IL, USA).

\section{Results}

3.1. Characteristics of the Study Participants. The participants included in the final analysis consisted of 1,144 individuals aged $68.3 \pm 3.1$ years (mean $\pm \mathrm{SD}$ ) with an equal gender distribution (male and female) and residence area (urban and rural) (see Table 1). A total of 784 (68.5\%) participants had dental caries, while 360 (31.5\%) did not. The details of the demographic characteristics, including the lifestyle, beliefs, and behaviors of the included participants, are shown in Table 1. 
TABLE 2: The prevalence of dental caries and the studied variables.

\begin{tabular}{|c|c|c|c|}
\hline Characteristic & $\begin{array}{c}\text { The number of participants } \\
\text { with caries }\end{array}$ & $\begin{array}{c}\text { The number of participants } \\
\text { without caries }\end{array}$ & ${ }^{\wedge} P$ value \\
\hline \multicolumn{4}{|c|}{ Demographic characteristics } \\
\hline \multicolumn{4}{|c|}{ Age } \\
\hline$<70$ & 494 & 248 & \\
\hline$\geq 70$ & 290 & 112 & $P=0.303$ \\
\hline \multicolumn{4}{|l|}{ Gender } \\
\hline Female, $n(\%)$ & 399 & 177 & \\
\hline Male, $n(\%)$ & 385 & 183 & $P=0.588$ \\
\hline \multicolumn{4}{|l|}{ Residency } \\
\hline Urban, $n(\%)$ & 419 & 154 & \\
\hline Rural, $n(\%)$ & 365 & 206 & $P=0.001$ \\
\hline \multicolumn{4}{|c|}{ Use of a removable upper jaw dental prosthesis } \\
\hline Yes, $n(\%)$ & 37 & 119 & \\
\hline No, $n(\%)$ & 747 & 241 & $P<0.001$ \\
\hline \multicolumn{4}{|c|}{ Use of a removable lower jaw dental prosthesis } \\
\hline Yes, $n(\%)$ & 23 & 100 & \\
\hline No, $n(\%)$ & 761 & 260 & $P<0.001$ \\
\hline \multicolumn{4}{|l|}{ Dental insurance } \\
\hline Yes, $n(\%)$ & 97 & 47 & \\
\hline No, $n(\%)$ & 688 & 312 & $P=0.745$ \\
\hline \multicolumn{4}{|l|}{ Number of true teeth } \\
\hline$>20$ & 537 & 193 & \\
\hline$\leq 20$ & 247 & 167 & $P<0.001$ \\
\hline \multicolumn{4}{|c|}{ Having toothache in previous years } \\
\hline Yes, $n(\%)$ & 417 & 118 & \\
\hline No, $n(\%)$ & 367 & 242 & $P<0.001$ \\
\hline \multicolumn{4}{|l|}{ Lifestyle } \\
\hline \multicolumn{4}{|l|}{ Smoking } \\
\hline Yes, $n(\%)$ & 538 & 199 & \\
\hline No, $n(\%)$ & 246 & 161 & $P<0.001$ \\
\hline \multicolumn{4}{|l|}{ Drinking alcohol } \\
\hline Yes, $n(\%)$ & 268 & 639 & \\
\hline No, $n(\%)$ & 92 & 145 & $P=0.006$ \\
\hline \multicolumn{4}{|l|}{ Eating candy frequently } \\
\hline Yes, $n(\%)$ & 42 & 30 & \\
\hline No, $n(\%)$ & 742 & 330 & $P=0.054$ \\
\hline \multicolumn{4}{|c|}{ Drinking carbonated beverages frequently } \\
\hline Yes, $n(\%)$ & 18 & 14 & \\
\hline No, $n(\%)$ & 766 & 346 & $P=0.129$ \\
\hline \multicolumn{4}{|l|}{ Domestic water access } \\
\hline Tap water, $n(\%)$ & 525 & 207 & \\
\hline No tap water, $n(\%)$ & 259 & 153 & $P=0.002$ \\
\hline \multicolumn{4}{|l|}{ Use of toothpick } \\
\hline Yes, $n(\%)$ & 468 & 252 & \\
\hline No, $n(\%)$ & 316 & 108 & $P=0.001$ \\
\hline
\end{tabular}


TABLE 2: Continued.

\begin{tabular}{|c|c|c|c|}
\hline Characteristic & $\begin{array}{c}\text { The number of participants } \\
\text { with caries }\end{array}$ & $\begin{array}{c}\text { The number of participants } \\
\text { without caries }\end{array}$ & ${ }^{\wedge} P$ value \\
\hline \multicolumn{4}{|l|}{ Use of dental floss } \\
\hline Yes, $n(\%)$ & 4 & 0 & \\
\hline No, $n(\%)$ & 780 & 360 & $P=0.175$ \\
\hline \multicolumn{4}{|c|}{ Use of fluoride toothpaste } \\
\hline Yes, $n(\%)$ & 242 & 96 & \\
\hline No, $n(\%)$ & 545 & 261 & $P=0.152$ \\
\hline \multicolumn{4}{|l|}{ Attitudes and behaviors } \\
\hline \multicolumn{4}{|c|}{ Healthcare service needs } \\
\hline Yes, $n(\%)$ & 531 & 218 & \\
\hline No, $n(\%)$ & 253 & 142 & $P=0.018$ \\
\hline \multicolumn{4}{|c|}{ Believing oral health has an impact on eating } \\
\hline Yes, $n(\%)$ & 474 & 167 & \\
\hline No, $n(\%)$ & 310 & 193 & $P<0.001$ \\
\hline \multicolumn{4}{|c|}{ Visiting dental clinic for caries without pain } \\
\hline Yes, $n(\%)$ & 206 & 90 & \\
\hline No, $n(\%)$ & 578 & 270 & $P=0.647$ \\
\hline \multicolumn{4}{|c|}{ Visiting dental clinic in the past year } \\
\hline Yes, $n(\%)$ & 132 & 61 & \\
\hline No, $n(\%)$ & 652 & 299 & $P=0.964$ \\
\hline \multicolumn{4}{|c|}{ Self-oral health assessment } \\
\hline Not good, $n(\%)$ & 289 & 158 & $P=0.024$ \\
\hline Good, $n(\%)$ & 495 & 202 & \\
\hline \multicolumn{4}{|c|}{ Self-oral hygiene assessment } \\
\hline Not good, $n(\%)$ & 199 & 59 & $P=0.001$ \\
\hline Good, $n(\%)$ & 585 & 301 & \\
\hline
\end{tabular}

${ }^{\wedge}$ Pearson's chi-square test.

3.2. Correlation Test for Each Target Factor. The correlation test results for each target factor (Appendix II) for the occurrence of dental caries in the participants are shown in Table 2. The univariable analysis showed that the targeted factors (e.g., residency, use of a removable upper jaw dental prosthesis, use of a removable lower jaw dental prosthesis, number of natural teeth, having toothache in previous years, smoking, drinking alcohol, domestic access to tap water, use of toothpicks, healthcare service needs, believing oral health has an impact on eating, self-oral health assessment, and self-oral hygiene assessment) had a statistical association with the occurrence of dental caries $(P<0.05)$. Other factors including gender, age, dental insurance, eating candy often, visiting the dental clinic for caries without pain, drinking carbonated beverages, use of dental floss and fluoride toothpaste, and visiting the dental clinic in the past year showed no statistical association (Table 2 ).

3.3. Multivariate Logistic Regression Results. The independent predictors for dental caries were identified based on multivariate logistics regression analysis (Table 3 ). In elderly individuals, a history of toothache in previous years (yes vs. no, $\mathrm{OR}=1.550,95 \% \mathrm{CI}: 1.164-2.063)$, use of a removable upper jaw dental prosthesis (yes vs. no, $\mathrm{OR}=4.320$ with 95\% CI: 2.647 to 7.051 ), use of a removable lower jaw dental prosthesis (yes vs. no, OR $=4.420$ with $95 \%$ CI from 2.477 to 7.885 ), living in a rural area (urban area vs. rural area, $\mathrm{OR}=0.676$ with $95 \% \mathrm{CI}$ from 0.503 to 0.908 ), smoking (yes vs. no, OR $=1.469,95 \% \mathrm{CI}: 1.084-1.992$ ), and drinking alcohol (yes vs. no, OR $=1.591$ with $95 \%$ CI from 1.130 to 2.240 ) were predictors for dental caries. On the other hand, good self-oral hygiene evaluation (good vs. not good, $\mathrm{OR}=0.606,95 \% \mathrm{CI}: 0.423-0.868)$ was a protective factor for participants against dental caries. We evaluated the potential collinearity between the variables, and the variance inflation factor (VIF) corresponding to these variables was less than 2, suggesting that there was no multicollinearity problem in the model.

3.4. Dental Caries Prediction Models. We randomly divided the data for the 1144 participants into two parts: $80 \%$ (915 cases) were included in the training sets for the establishment of unconditional logistic regression and the GRNN early warning model, while the remaining 20\% (229 cases) were used as test sets for model prediction (see Figure 1). The data collected from the training set $(80 \%, 915$ cases) were tested 
TABLE 3: The independent predictors according to the multivariate logistic analysis.

\begin{tabular}{lcr}
\hline Characteristic & OR (95\% confidence interval) & $P$ value \\
\hline Resident area & $0.676(0.503-0.908)$ & $P=0.009$ \\
$\quad$ Urban vs. rural & $4.320(2.647-7.051)$ & $P<0.001$ \\
Use of a removable upper jaw dental prosthesis & \\
$\quad$ Yes vs. no & $4.420(2.477-7.885)$ \\
Use of a removable lower jaw dental prosthesis & $1.550(1.164-2.063)$ \\
$\quad$ Yes vs. no & \\
Having toothache in previous years & $1.469(1.084-1.992)$ \\
$\quad$ Yes vs. no & \\
Smoking & $1.591(1.130-2.240)$ \\
$\quad$ Yes vs. no & \\
Drinking alcohol & \\
$\quad$ Yes vs. no & \\
Self-oral hygiene assessment & \\
$\quad$ Good vs. not good & $0.606(0.423-0.868)$ \\
\hline
\end{tabular}

Abbreviation: OR for odds ratio.

TABLE 4: The independent predictors according to the unconditional multivariate logistic regression.

\begin{tabular}{|c|c|c|}
\hline Characteristic & OR (95\% confidence interval) & $P$ value \\
\hline \multicolumn{3}{|l|}{ Resident area } \\
\hline Urban vs. rural & $0.656(0.475-0.906)$ & $P=0.011$ \\
\hline Number of cigarettes per day & $0.652(0.443-0.957)$ & $P=0.029$ \\
\hline \multicolumn{3}{|c|}{ Use of a removable upper jaw dental prosthesis } \\
\hline Yes vs. no & $5.655(3.242-9.864)$ & $P<0.001$ \\
\hline \multicolumn{3}{|c|}{ Use of a removable lower jaw dental prosthesis } \\
\hline Yes vs. no & $5.808(3.025-11.152)$ & $P<0.001$ \\
\hline \multicolumn{3}{|l|}{ Having toothache in previous years } \\
\hline Yes vs. no & $1.628(1.174-2.256)$ & $P=0.003$ \\
\hline \multicolumn{3}{|c|}{ Drinking carbonated beverages frequently } \\
\hline Yes vs. no & $0.270(0.113-0.644)$ & $P=0.003$ \\
\hline \multicolumn{3}{|l|}{ Drinking alcohol } \\
\hline Yes vs. no & $0.523(0.359-0.760)$ & $P=0.001$ \\
\hline
\end{tabular}

Abbreviation: OR for odds ratio.

with a one-way chi-square test, and 15 variables were found to be related to the occurrence of dental caries in the elderly population (Appendix I). These variables included the residence area $(P=0.002)$, upper and lower removable dental prosthesis $(P<0.001)$, number of true teeth and having toothache in previous years $(P<0.001)$, smoking $(P<0.001)$, number of cigarettes per day $(P=0.003)$, drinking alcohol $(P=0.006)$, eating candy frequently $(P=0.0034)$, drinking carbonated beverages frequently $(P=0.023)$, domestic water access $(P=0.005)$, use of toothpicks $(P=0.004)$, believing that oral health has an impact on eating $(P=0.001)$, self-oral health assessment $(P=0.007)$, and self-oral hygiene assessment $(P=0.001)$.

According to the unconditional multivariate logistic regression, seven variables (residence area, number of cigarettes/day, using a removable upper/lower jaw dental prosthe-
TABLE 5: The fitting and prediction results of the logistic regression model for the subjects in the training and test sets.

\begin{tabular}{lcccc}
\hline $\begin{array}{l}\text { Predictive } \\
\text { results }\end{array}$ & $\begin{array}{c}\text { Training set } \\
\text { Number of } \\
\text { true caries }\end{array}$ & $\begin{array}{c}\text { Number of } \\
\text { true no } \\
\text { caries }\end{array}$ & $\begin{array}{c}\text { Number of } \\
\text { true caries }\end{array}$ & $\begin{array}{c}\text { Number of } \\
\text { true no } \\
\text { caries }\end{array}$ \\
\hline+ & 565 & 151 & 131 & 51 \\
- & 64 & 135 & 24 & 23 \\
Total & 629 & 286 & 155 & 74 \\
\hline
\end{tabular}

sis, toothache in previous years, and drinking carbonated beverages and alcohol) were entered into the model (Table 4). The fitting and prediction results for the model using the subjects in the training and test sets (229 cases) are compared in Table 5 . The results showed an optimal 


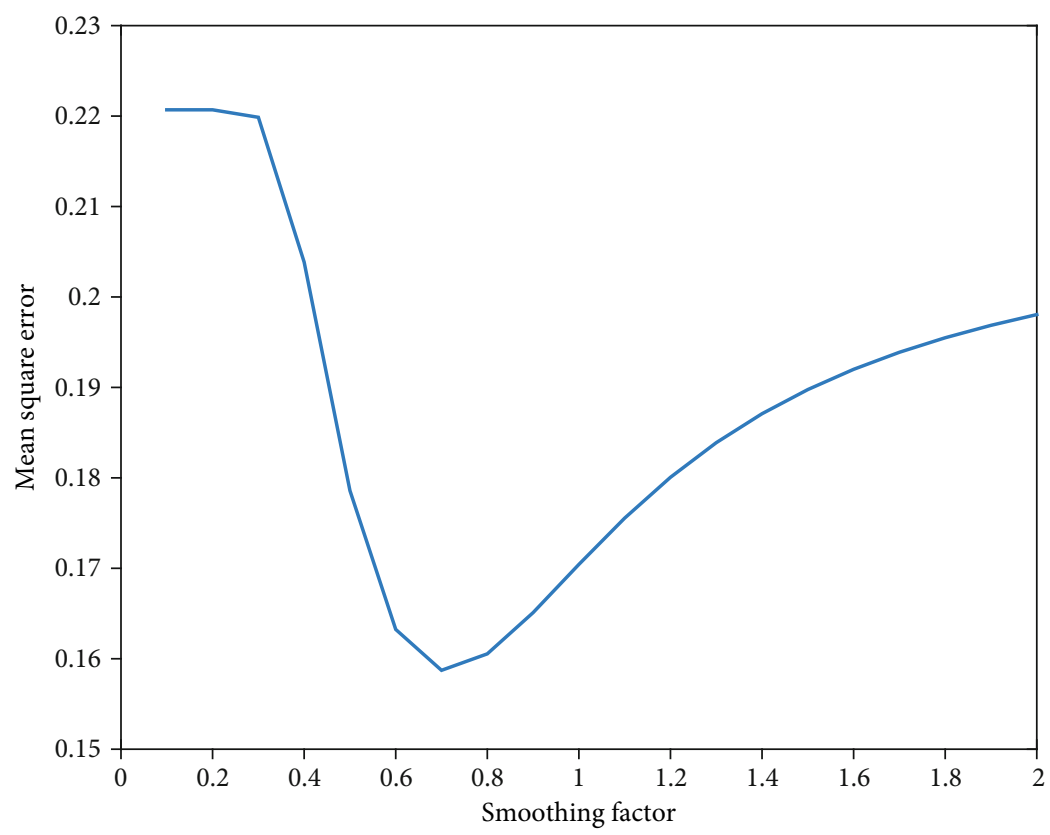

FIGURE 2: Determination of mean square error and optimal smoothing factor.

smoothing factor of 0.7 (Figure 2). Seven statistically significant variables from the chi-square test were used as the input, and the outcome variable (the presence of caries in elderly participants) was used as the output. The fitting and prediction results for the GRNN model using the subjects in the training and test sets are shown in Table 6.

3.5. Comparison between the Unconditional Logistic Regression and GRNN Models. A comparison of the accuracy of the unconditional logistic regression and GRNN models showed that the classification consistency, sensitivity, and specificity of the GRNN model were higher than those of the unconditional logistic regression model (Table 7).

The optimal cut-off value for the predictive probability of the logistic regression model in the present study was found to be 0.606 , with a corresponding Youden's index of 0.370 . The optimal cut-off value for the GRNN model's predictive probability was 0.680 , with a corresponding Youden's index of 0.638. The ROC curves for the logistic regression and GRNN models generated using the data set with the best cut-off values are shown in Figure 3. The areas under the ROC curves for the logistic regression and GRNN models were 0.578 and 0.777 , respectively, with corresponding $P$ values of 0.056 and $<0.001$ compared to the baseline. The $P$ value for the comparison of the area under the ROC curve for the two models was $<0.001$. The GRNN model was better than the unconditional logistic regression model in terms of accuracy.

\section{Discussion}

In the present study, a GRNN predicting model was used for dental caries risk assessment among geriatric residents. For this purpose, we investigated 1144 old (65-74 years)
TABLE 6: The fitting and prediction results of the generalized regression neural network (GRNN) model for the subjects in the training and test sets.

\begin{tabular}{lcccc}
\hline $\begin{array}{l}\text { Predictive } \\
\text { results }\end{array}$ & $\begin{array}{c}\text { Training set } \\
\text { Number of } \\
\text { true caries }\end{array}$ & $\begin{array}{c}\text { Number of } \\
\text { true no } \\
\text { caries }\end{array}$ & $\begin{array}{c}\text { Tumber of set } \\
\text { true caries }\end{array}$ & $\begin{array}{c}\text { Number of } \\
\text { true no } \\
\text { caries }\end{array}$ \\
\hline+ & 575 & 79 & 132 & 22 \\
- & 54 & 207 & 23 & 52 \\
Total & 629 & 286 & 155 & 74 \\
\hline
\end{tabular}

residents of Liaoning province, China, and performed a comprehensive statistical analysis comparing the accuracy of unconditional logistic regression and GRNN models. The caries rate in the geriatric population was $68.5 \%$, which is similar to the survey results from the third oral health epidemiological investigation in the Liaoning province performed 11 years ago [12]. These findings suggested that oral healthcare for the old residents of this province had not received enough attention and suffered from a lack of corresponding oral healthcare publicity and education in recent decades. There are a number of factors associated with compromised oral healthcare in the region. For example, although the living standards have improved in recent years, the eating habits of elderly individuals have not changed. Our results showed that the use of dental floss (0.3\%) and fluoride toothpaste (29.5\%) and the proportion of participants who sought medical treatment $(27.0 \%)$ for oral diseases were remarkably low. Lack of patient education and understanding of oral health management also contributed to poor oral healthcare. The financial burden is also an essential factor in 
TABLE 7: Comparison of the accuracy between the unconditional logistic regression model and the generalized regression neural network (GRNN) model.

\begin{tabular}{|c|c|c|c|c|c|c|}
\hline \multirow[t]{2}{*}{ Models } & \multicolumn{3}{|c|}{ Training set } & \multicolumn{3}{|c|}{ Test set } \\
\hline & Consistency & Sensitivity & Specificity & Consistency & Sensitivity & Specificity \\
\hline Logistic & $76.50 \%$ & $89.83 \%$ & $47.20 \%$ & $67.25 \%$ & $84.52 \%$ & $31.08 \%$ \\
\hline GRNN & $85.46 \%$ & $91.41 \%$ & $72.38 \%$ & $77.29 \%$ & $85.16 \%$ & $70.27 \%$ \\
\hline$\chi^{2}$ & 21.888 & 0.988 & 57.284 & 11.758 & 0.000 & 25.290 \\
\hline$P$ value & 0.001 & 0.320 & 0.001 & 0.001 & 1.000 & 0.001 \\
\hline
\end{tabular}

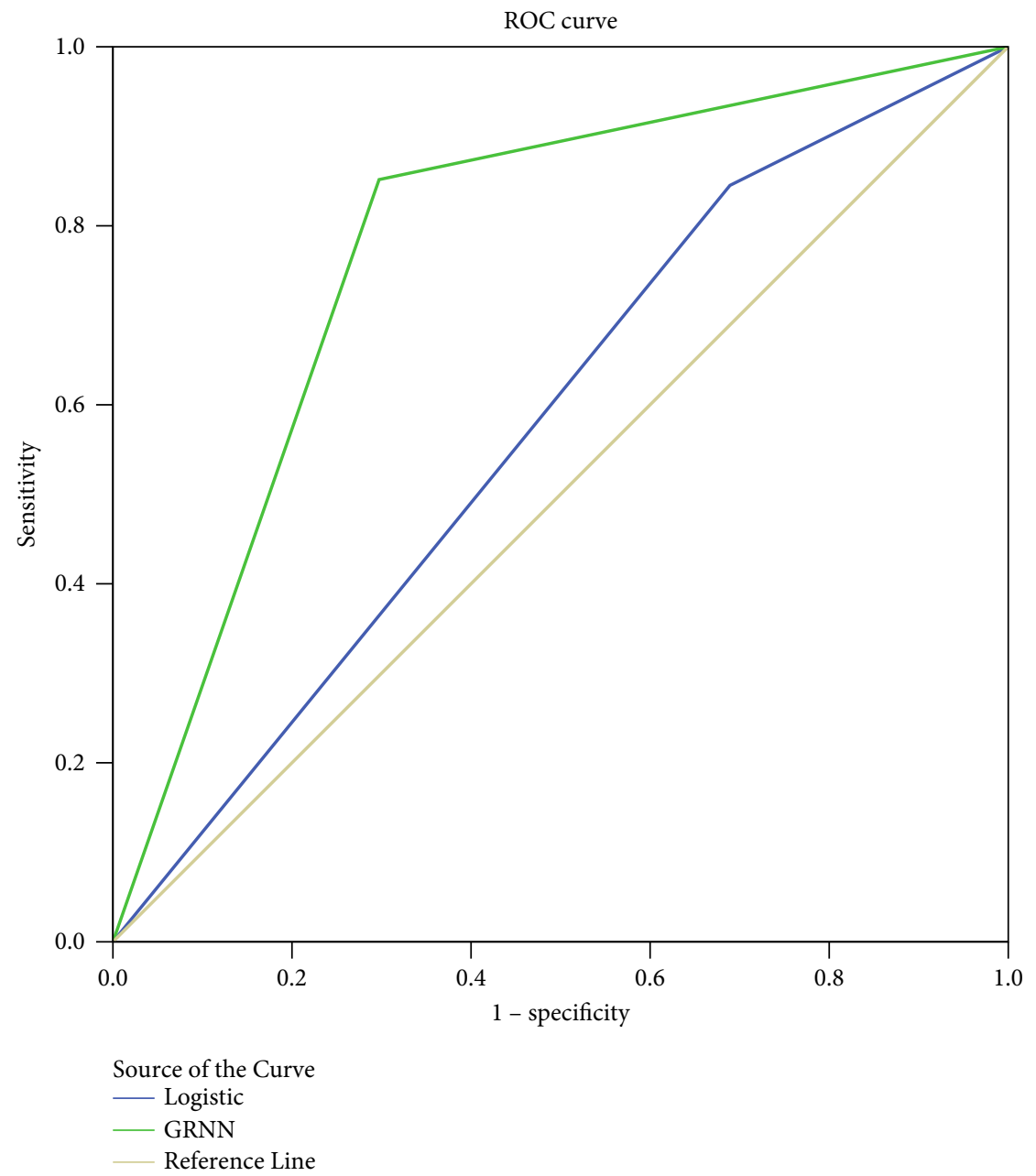

FIGURE 3: ROC curves for the logistic regression and GRNN models in the data set with the best cut-off values.

the development of caries as most elderly residents in the Liaoning province have to pay for oral healthcare services $[14,20]$.

The present study showed that the main risk factors associated with elderly dental caries were toothache history, residence type, and smoking and drinking status. These factors were slightly different from those identified in the $3^{\text {rd }}$ oral health epidemiological investigation, which indicated that using fluoride toothpaste, consuming sugary foods, frequency of teeth brushing, and the area of residence are factors related to dental caries that may affect the Chinese geriatric population (65-74 years) [12]. In contrast, we did not find a statistical significance in using fluoride toothpaste and eating sugar-containing foods with elderly dental caries. This may be due to the low utilization of fluoride toothpaste and sugar-containing foods in the studied population; only $29.5 \%$ (338/1144) used fluoride-containing toothpaste, and only a few participants were able to eat sugary desserts or beverages on a daily basis.

Smoking and drinking are common risk factors for several chronic oral conditions, including gingivitis, 
periodontitis, and gingival recession among elderly dental patients [24]. Plaque adherence to the cementoenamel junction and root surface can induce changes in the cementum, leading to demineralization, decay, and root caries [25]. The synergistic effects of drinking and smoking are also likely to increase the risk of oral diseases in elderly individuals, further enhancing the occurrence and development of dental caries [20].

Using removable dental prosthesis in the upper jaw (OR (95\% CI), 4.320 (2.647-7.051)) and lower jaw (OR (95\% CI), 4.420 (2.477-7.885)) had a significant influence on the occurrence of dental caries in the elderly participants. These findings are in agreement with previous studies [1,26-28]. The use of a removable prosthesis may facilitate the growth of cariogenic bacteria by changing the oral environment, increasing the risk of developing dental caries [28, 29]. Therefore, for patients who wear removable dental prosthesis, dental healthcare professionals should be alert for the possibility of dental caries and take measures to prolong the survival of teeth.

Another important risk factor associated with the occurrence of dental caries in the elderly population is self-oral hygiene assessment. A better capability to assess self-oral hygiene resulted in the reduction of dental caries $(P=0.005)$. Patients with good oral hygiene are confident in their self-oral hygiene assessment [30].

The present study found that participants with a history of toothache were more susceptible to dental caries, which is consistent with the findings reported by Liu et al. [31]. Elderly patients suffering from toothache usually endure pain rather than visiting the dentist to avoid the inconvenience or due to economic factors. Over an extended period, such an attitude may lead to the development of dental caries and associated dental pain [32]. Living in rural areas was a protective factor for elderly caries, which is consistent with previous studies [33, 34]. With the rapid development of urban economies, various factors such as the living standards, eating habits, and sugar consumption of urban elderly residents have become remarkably different from those of the residents of rural areas. For instance, the type of food intake is more diverse than that in rural areas, and most consumed foods are finely processed products. Consequently, the daily dietary sugar intake of urban elderly residents is higher than that of rural residents. Additionally, rural residents in this province usually like to eat dietary fiber-rich foods. Nutritional fiber-rich foods have an oral self-cleaning effect, thus protecting against the development of caries [35, 36].

GRNN was applied in the present study to establish a prediction model for dental caries in the elderly population. Compared to the traditional BPNN, the training process for GRNN does not need to be iterative, and the training parameters for the network only have an optimal smoothing factor [37]. After the optimal smoothing factor is determined, the predictive ability is stable. In the present study, $20 \%$ of the training set was randomly selected as the test set to find the optimal smoothing factor for the generalized regression neural network, and good extrapolation of the prediction results was ensured. In terms of variable selection, we first used the single-factor chi-square test to reduce the dimensions of modeling variables to ensure the simplicity of the model and prevent the existence of too much redundant information from reducing the prediction effects.

In the present study, we found that the consistency, sensitivity, and specificity of the GRNN model were better than those of the traditional unconditional multivariate logistic regression model in the training and test sets. The area under the ROC curve for the GRNN model was also larger than that of the unconditional multivariate logistic regression model, with better prediction results. However, the shortcomings of the GRNN model were also apparent. According to the OR value obtained in the unconditional multivariate logistic regression model, we can explain the results professionally and roughly judge the degree of influence from the variables on the results, which is not possible using a GRNN model. GRNN acts like a "black box," which makes it difficult for us to interpret the results from a professional point of view and to judge the contribution of variables. GRNN has no way to calculate the $P$ and $\mathrm{OR}$ values for each included variable, so there is no way to determine the contribution of GRNN or to explain the risk of disease. Further, GRNN can only provide the results of disease prediction. GRNN can predict diseases, but it is impossible to determine which of the variables included in the prediction model play a key role. Therefore, compared with traditional methods, the GRNN model is more accurate for prediction, but is less explanatory. This is common for all in-depth learning models. Another limitation of the current cross-sectional study is that the factors used to create the GRNN model may not be accurate predictors as the study is not a cohort study. Additionally, reading limitations for patients may have introduced bias while they completed the questionnaire. Future research shall focus on patient follow-up and other important factors (e.g., systematic diseases) to assess the validity of the tool.

\section{Conclusion}

The geriatric (65-74 years) residents of Liaoning, China, presented a high rate of dental caries. Residents with a history of toothache and smoking habits were more susceptible to dental caries. The GRNN model had better consistency, sensitivity, and specificity than traditional unconditional multifactor logistic regression models in both the training and test sets. Therefore, the GRNN early warning model is an accurate and meaningful tool for screening, early diagnosis, and treatment planning for elderly individuals with a high risk of dental caries. Simultaneously, further measures should be taken to promote oral healthcare education for the elderly population and establish a sound primary oral healthcare system.

\section{Abbreviations}

ANN: Artificial neural network

GRNN: Generalized regression neural network 
BPNN: Back-propagation neural network

FNN: Fuzzy neural network

PNN: Probabilistic neural network.

\section{Data Availability}

The datasets used and/or analyzed during the current study are available from the corresponding author on reasonable request.

\section{Ethical Approval}

This study was approved by the Ethics Committee of the School and Hospital of Stomatology, China Medical University, Liaoning Provincial Key Laboratory of Oral Diseases. All procedures performed in studies involving human participants were in accordance with the ethical standards of the institutional and national research committee and with the 1964 Helsinki declaration and its later amendments or comparable ethical standards.

\section{Consent}

All data published here are under consent for publication. Written informed consent was obtained from all individual participants included in the study.

\section{Disclosure}

The authors declare that they have no financial relationship with the organization that sponsored the research, and the funding body was not involved in study design, data collection, analysis, and writing of the study.

\section{Conflicts of Interest}

The authors declare that they have no competing interests.

\section{Authors' Contributions}

LL and ZHY designed the study. LL, WW, SYZ, KQZ, JL, YL, and ZHY collected the data. WW and LL analyzed the data. LL drafted and wrote the manuscript. LL, ZHY, and WW revised the manuscript critically for intellectual content. All authors read and approved the final version of the manuscript.

\section{Acknowledgments}

We sincerely thank all the guardians for their participation. This study was supported by a grant from the National Natural Science Foundation of China (No. 81400571).

\section{Supplementary Materials}

Supplementary 1. Appendix I: variables related to the occurrence of dental caries in elderly individuals according to the training set data.

Supplementary 2. Appendix II: the full list of variables assessed in the present study.

\section{References}

[1] X. Chen, J. J. Clark, J. S. Preisser, S. Naorungroj, and S. K. Shuman, "Dental caries in older adults in the last year of life," Journal of the American Geriatrics Society, vol. 61, no. 8, pp. 1345-1350, 2013.

[2] R. C. Castrejon-Perez, S. A. Borges-Yanez, L. M. GutierrezRobledo, and J. A. Avila-Funes, "Oral health conditions and frailty in Mexican community-dwelling elderly: a cross sectional analysis," BMC Public Health, vol. 12, no. 1, 2012.

[3] R. Marino, M. Morgan, A. Kiyak, E. Schwarz, and S. Naqvi, "Oral health in a convenience sample of Chinese older adults living in Melbourne, Australia," International Journal of Public Health, vol. 57, no. 2, pp. 383-390, 2012.

[4] R. Srivastava, B. Nongkynrih, V. P. Mathur, A. Goswami, and S. K. Gupta, "High burden of dental caries in geriatric population of India: a systematic review," Indian Journal of Public Health, vol. 56, no. 2, pp. 129-132, 2012.

[5] X. Chen, J. J. J. Clark, and S. Naorungroj, "Length of tooth survival in older adults with complex medical, functional and dental backgrounds," J Am Dent Assoc, vol. 143, no. 6, pp. 566-578, 2012.

[6] D. L. Huang, K. C. G. Chan, and B. A. Young, "Poor oral health and quality of life in older U.S. adults with diabetes mellitus," Journal of the American Geriatrics Society, vol. 61, no. 10, pp. 1782-1788, 2013.

[7] K. Solemdal, L. Sandvik, C. Moinichen-Berstad, K. Skog, T. Willumsen, and M. Mowe, "Association between oral health and body cell mass in hospitalised elderly," Gerodontology, vol. 29, no. 2, pp. e1038-e1044, 2012.

[8] F. DeStefano, R. F. Anda, H. S. Kahn, D. F. Williamson, and C. M. Russell, "Dental disease and risk of coronary heart disease and mortality," BMJ, vol. 306, no. 6879, pp. 688-691, 1993.

[9] H. Carl and M. K. Mary, World Population Data Sheet, Population Reference Bureau, Washington, DC, 2007.

[10] D. Hu, X. Hong, and X. Li, "Oral health in China-trends and challenges," International Journal of Oral Science, vol. 3, no. 1, pp. 7-12, 2011.

[11] X. Wang, The Fourth National Oral Health Epidemiological Survey Report, People's Medical Publishing House, Peking, 2018.

[12] X. Qi, Report of the third national oral health survey in China, People's Medical Publishing House, Beijing, China, 2008.

[13] B. A. Burt, "Concepts of risk in dental public health," Community Dentistry and Oral Epidemiology, vol. 33, no. 4, pp. 240247, 2005.

[14] L. Liu, Y. Zhang, W. Wu, and R. Cheng, "Characteristics of dental care-seeking behavior and related sociodemographic factors in a middle-aged and elderly population in Northeast China," BMC Oral Health, vol. 15, no. 1, 2015.

[15] J. Zou, Y. Han, and S. S. So, "Overview of artificial neural networks," Methods in Molecular Biology, vol. 458, pp. 15-23, 2008.

[16] M. Huang and D. Wang, "Application of artificial neural network in stomatology," Journal of Dental Prevention and Treatment, vol. 17, p. 4, 2009.

[17] A. J. Al-Mahasneh, S. G. Anavatti, and M. A. Garratt, "Review of applications of generalized regression neural networks in identification and control of dynamic systems," 2018, http:// arxiv.org/abs/180511236. 
[18] D. M. Pelt and K. J. Batenburg, "Fast tomographic reconstruction from limited data using artificial neural networks," IEEE transactions on image processing: a publication of the IEEE Signal Processing Society, vol. 22, no. 12, pp. 5238-5251, 2013.

[19] H. Zakrzewska, A. Machoy-Mokrzyńska, M. Materny, I. Gutowska, and Z. Machoy, "Estimation of fluoride distribution in the mandible and teeth of the red deer (Cervus elaphus L.) from industrially polluted areas in Poland," Archives of Oral Biology, vol. 50, no. 3, pp. 309-316, 2005.

[20] D. Hu, Oral Prevention Medicine, People's Health Publishing House, Pejing, 2013.

[21] L. Liu, Y. Zhang, W. Wu, M. Cheng, Y. Li, and R. Cheng, "Prevalence and correlates of dental caries in an elderly population in Northeast China," PLoS One, vol. 8, no. 11, article e78723, 2013.

[22] World Health Organization, Oral health surveys: basic methods, World Health Organization, Geneva, 2013.

[23] D. F. Specht, “A general regression neural network," IEEE Transactions on Neural Networks, vol. 2, no. 6, pp. 568-576, 1991.

[24] J. D. Glover, D. M. S. Hetzel, and S. K. Tennant, "The socioeconomic gradient and chronic illness and associated risk factors in Australia," Australia and New Zealand Health Policy, vol. 1, no. 1, p. 8, 2004.

[25] S. Kularatne and L. Ekanayake, "Root surface caries in older individuals from Sri Lanka," Caries Research, vol. 41, no. 4, pp. 252-256, 2007.

[26] R. H. Saunders Jr. and C. Meyerowitz, "Dental caries in older adults," Dental Clinics of North America, vol. 49, no. 2, pp. 293-308, 2005.

[27] N. J. A. Jepson, P. J. Moynihan, P. J. Kelly, G. W. Watson, and J. M. Thomason, "Caries incidence following restoration of shortened lower dental arches in a randomized controlled trial," British Dental Journal, vol. 191, no. 3, pp. 140-144, 2001.

[28] J. Tanaka, M. Tanaka, and T. Kawazoe, "Longitudinal research on the oral environment of elderly wearing fixed or removable prostheses," Journal of Prosthodontic Research, vol. 53, no. 2, pp. 83-88, 2009.

[29] P. M. Preshaw, A. W. G. Walls, N. S. Jakubovics, P. J. Moynihan, N. J. A. Jepson, and Z. Loewy, "Association of removable partial denture use with oral and systemic health," Journal of Dentistry, vol. 39, no. 11, pp. 711-719, 2011.

[30] P. E. Petersen, J. Aleksejuniene, L. B. Christensen, H. M. Eriksen, and I. Kalo, "Oral health behavior and attitudes of adults in Lithuania," Acta Odontologica Scandinavica, vol. 58, pp. 243-248, 2009.

[31] X. Liu, D. Huang, Z. Wang, L. Zhang, and X. Wang, "Analysis of related factors in the elderly crown caries of permanent teeth," China Health Standards Management, vol. 7, p. 2, 2016.

[32] L. R. A. Noro, A. G. Roncalli, F. I. R. M. Júnior, K. C. de Lima, and A. K. M. Teixeira, "Toothache and social and economic conditions among adolescents in northeastern Brazil," Ciência \& Saúde Coletiva, vol. 19, no. 1, pp. 105-114, 2014.

[33] M. Ueno, S. Ohara, M. Inoue, S. Tsugane, and Y. Kawaguchi, "Association between education level and dentition status in Japanese adults: Japan public health center-based oral health study," Community Dentistry and Oral Epidemiology, vol. 40, no. 6, pp. 481-487, 2012.

[34] B. M. Henriksen, T. Axell, and K. Laake, "Geographic differences in tooth loss and denture-wearing among the elderly in
Norway," Community Dentistry and Oral Epidemiology, vol. 31, no. 6, pp. 403-411, 2003.

[35] W. Lin, G. Si, H. Li, B. Chen, and X. Zeng, "Investigation and analysis of the oral health status of 359 residents of Baikuyao nationality in Guangxi, China," Journal of Guangxi Medical University, vol. 31, p. 4, 2014.

[36] W. Sun, "Investigation on oral health status of Mongolian and Han nationality population in Siziwang banner," Guide of China Medicine, vol. 11, p. 2, 2013.

[37] T. Manning, R. D. Sleator, and P. Walsh, "Biologically inspired intelligent decision making: a commentary on the use of artificial neural networks in bioinformatics," Bioengineered, vol. 5, no. 2, pp. 80-95, 2014. 